\title{
Programmed Cell Death (PCD) Regulation Related to Development in Plant
}

\author{
S. Dogra ", G. Chand, B. K. Sinha and M. Banoo \\ Department of Plant Physiology, Sher-e-KashmirUniversity of Agricultural Sciences \& \\ Technology Jammu, J\&K, 180009, India
}

*Corresponding author

\begin{tabular}{|l|}
\hline Ke y w or d s \\
Programmed cell \\
death, \\
Developmental \\
PCD, Reactive \\
oxygen species
\end{tabular}

\section{A B S T R A C T}

Programmed cell death (PCD) is a fundamental cellular process that has adopted a plethora of vital functions in multicellular organisms. Such phenomenon is associated with recovery of cellular compounds and sustaining plant life. Basic morphological and biochemical features of PCD are believed to be conserved in both plants and animals. Nevertheless, recent studies demonstrate an involvement of organelles such as vacuole and chloroplast in plant cell death regulation, indicating that plants evolved own cell death machinery. The importance of understanding PCD signaling pathways, with their elicitors and effectors, is in order to improve plant productivity and resistance to environmental stresses. This article describes some of the fundamental characteristics of plant $\mathrm{dPCD}$ and raises points that may lead to a better understanding and novel strategies for plant molecular breeding.

\section{Introduction}

Programmed cell death (PCD) is a genetically regulated process responsible for the elimination of undesirable cells in eukaryotes; necessary for development and survival. Programmed cell death (PCD) is a physiological cell death process involved in the selective elimination of unwanted cells. Cells that are damaged and unable to function correctly can also undergo PCD. This removes potentially harmful cells and prevents them from multiplying and spreading. PCD process leads to the active participation of the dying cells, and could be regulated by genetically controlled, well-orchestrated cell suicide machinery. The common process in such instances comprise one or more phenotypes such as cytoplasmic shrinkage, membrane blebbing, loss of cell-to-cell contact, DNA fragmentation, cytochrome $\mathrm{c}$ release from mitochondria, cell shrinkage, generation of reactive oxygen species, exposure of phosphatidylserine, etc. (Bonneau et al., 
2008).Ample evidence can be presented to support that cell death during plant development and environmental challenges involves PCD (Figure 1).

\section{PCD events in plants}

In plants, various forms of PCD have been described as an inherent part of development, as well as a response to biotic and abiotic stresses. In plants, different types of PCD play crucial roles in vegetative and reproductive development (dPCD), as well as in the reaction to environmental stresses (ePCD. Various dPCD events can be distinguished on the basis of their developmental context. Differentiation-induced dPCD occurs as an inherent final differentiation step of particular cell types, e.g., xylem, anther tapetum, or root cap cells. Some cell types, however, can initiate $\mathrm{dPCD}$ in a facultative fashion, for instance, as a result of cell-to-cell signaling during self-incompatibility responses or on the basis of positional information during aerenchyma formation or leaf perforation (Gunawardena, 2008). Finally, age-induced dPCD occurs in all cell types of organs or even entire organisms as the end point of plant senescence.

\section{Cell death in reproductive and vegetative development}

Plants eliminate cells, organs and parts during various developmental processes

Sexual reproduction in plants is important for population survival as well as for increasing genetic diversity. During gametophyte formation, fertilization, and seed development, there are numerous instances of developmentally regulated cell elimination, many of which are forms of dPCD essential for successful plant reproduction. After germination, PCD processes continue to play important roles during vegetative plant development. In flowering plants (angiosperms), the gametophytes are reduced to a few cells developing inside the sporophyte. Female gametophyte (FG) development in the ovule and male gametophyte (pollen) formation in the anther are sustained by adjacent sporophytic tissues. A number of $\mathrm{dPCD}$ processes are required for successful gametophyte development (Fig. 2).

\section{Fertilization}

After landing on the floral stigma, pollen grains germinate pollen tubes (PTs) via which the sperm cells are transported to the FG to accomplish double fertilization. These processes require the elimination of several cells in tightly controlled ways.

Self-incompatibility (SI) is a genetically controlled process by which many flowering plants prevent self-fertilization and inbreeding. SI displays several PCD features, including actin depolymerization, ROS signaling, caspase-like protease activities, and DNA fragmentation. Transmitting tract cell death: In many angiosperms, the transmitting tract (TT) is a specialized tissue that guides and supports PT growth toward the ovule (Figure 3). In some species, TT cell death is thought to be important for supporting growing PTs.

\section{Seed Development}

After fertilization, the development of the seed coordinates growth and differentiation of the embryo proper, embryonic suspensor, endosperm, and seed coat. After germination, only the embryo proper will have survived; all other seed tissues will have died at specific time points of seed development. The embryonic suspensor anchors the embryo in the seed cavity and is thought to contribute to nutrient transfer during early embryo development (Figure 4). Subsequently, in 
many plant species the suspensor is eliminated via vacuolar PCD (Smertenko and Bozhkov, 2014).

Seed coat cell death: The seed integument cell layers undergo PCD in a specific order, and their fused cell corpses form a protective seed coat termed testa. In A. thaliana, integument PCD is delayed in mutants, and this cysteine protease is specifically expressed in two integument layers before PCD. The endosperm is the second zygotic product generated by double fertilization, but in contrast to the embryo, it is but a transient nutritive tissue. In cereals, the starchy endosperm (SE) undergoes a nonlytic PCD after seed filling. The outermost endosperm layer, the aleurone, survives seed development and dies only during germination (LopezFernandez and Maldonado, 2015).

\section{Xylogenesis}

The xylem is a structure and transport tissue distributing water and solutes from the roots to the aerial organs and providing mechanical support to the plant body. PCD of xylem tracheids and tracheary elements (TEs) culminates in protoplast clearance, producing interconnected hollow cell corpses with reinforced cell walls (Figure 5) (Menard and Pesquet, 2015). Studies have revealed that this cell death is under spatial and temporal regulation. In this system single mesophyll cells isolated from Zinnia leaves transdifferentiate synchronously into tracheary elements at a high frequency without cell division. Various hormones, including brassinosteroids and ethylene, initiate TE differentiation and dPCD.

\section{Lateral root cap differentiation}

The root cap is an organ that unsheathes the root tip and has important functions for root growth, gravity sensing, and root system architecture. In $A$. thaliana, the root cap consists of a central columella and a peripheral lateral root cap (LRC). The constant size and location of the LRC at the growing root tip are maintained by rapid cellular turnover. The continuous generation of new LRC layers by specific stem cells is counterbalanced by tightly controlled dPCD that eliminates cells at the root cap edge (Figure 5) (Fendrych et al., 2014).

\section{Aerenchyma formation}

Formation of air-conducting aerenchyma tissue facilitates a plant's survival in lowoxygen soils. The formation of lysigenous aerenchyma mediated by PCD (Figure 6) occurs constitutively in wetland species and can be induced by oxygen deprivation in waterlogged plants.In the root cortex of maize subjected to hypoxia, ethylene induces lysigenous aerenchyma formation by activating genes related to cell wall degradation and PCD.

\section{Leaf Morphogenesis}

Although leaf morphogenesis relies mainly on differential growth, specific leaf shapes require cell elimination. PCD causes leaf perforation in lace plant (Aponogeton madagascariensis) and in a few aroids (Figure $6)$.

Perforation PCD occurs between the leaf veins, creating the fenestrate pattern of mature lace plant leaves. Tonoplast rupture is the first evident PCD feature to occur, followed by DNA fragmentation, changes in nuclear morphology, cytoplasmic shrinkage, and organelle dismantling.

Interestingly, inhibition of ethylene biosynthesis or ethylene perception leads to a significant reduction in the number of leaf perforations in the lace plant (Dauphinee et al., 2012). 


\section{Cell death as the end point of senescence}

Plant senescence controls nutrient remobilization during stress- or age-induced degeneration of tissues, organs, or entire organisms, concluding the life cycle of monocarpic plant species (Figure 7) (MunneBosch, 2015). Cell death marks the final stage of plant senescence. Senescence in plants can refer to at least two distinct processes: The aging of various tissues and organs as the whole plant matures and the process of the whole plant death that sometimes occurs after fertilization and called as monocarpic senescence. Senescence is a genetically controlled developmental process, which is internally programmed. Ultrastructural researches showed that some features of senescence resemble to the typical markers of PCD.

Leaf senescence is driven by transcriptome reprogramming and is influenced by multiple exogenous and endogenous cues (Schippers, 2015). Ethylene promotes senescence, and mutant's defective in its biosynthesis, perception, or signaling display increased leaf longevity (Koyama, 2014).

\section{Future Perspectives}

The process of PCD is essential for ensuring the proper development of plants as well as ensuring a robust defence response against invading pathogens. $\mathrm{dPCD}$ is essential for successful development and growth of complex multicellular organisms.PCD is needed to destroy the cells that represent a threat to the integrity of the organism. There is also common evidence of transcriptional regulation, though within different contexts. In many dPCD forms, cells need to gradually acquire a competence to execute cell death upon specific developmental signals. But major contributions will come from research focused specifically on how cell death occurs with in unique biological contexts (Hypersensitive response, etc.) that are of interest to plant biologists and important for improving agriculture.

Fig.1 PCD is involved in many phases through vegetative and reproductive development and response to environmental stresses

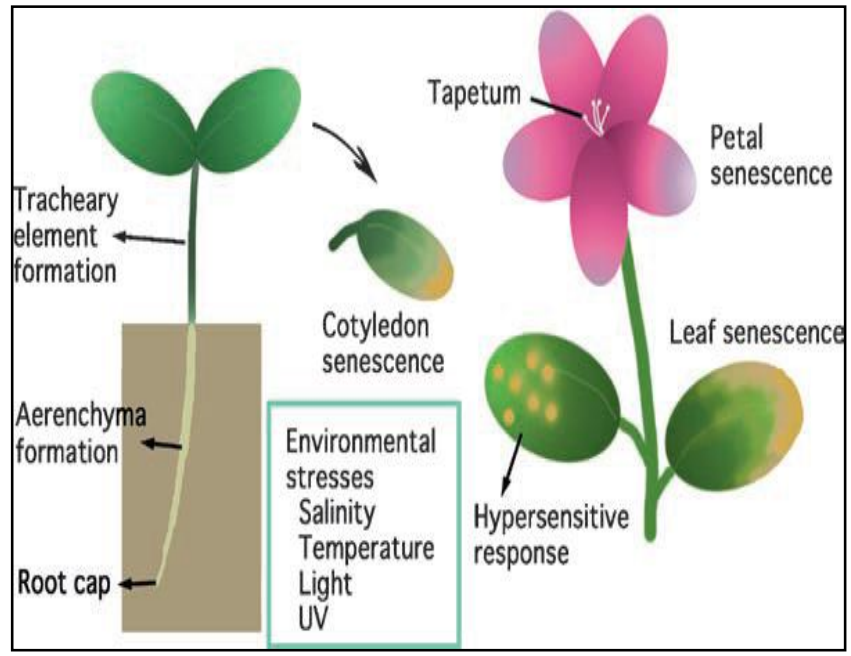


Fig.2 PCD during (a)Non-functional megaspores undergo cell death during functional megaspore selection. (b) Nucleus degradation during female gametophyte development. (c) Degradation of the tapetal layer. Dying cells or tissues are marked in purple and dead cells or tissues are in black

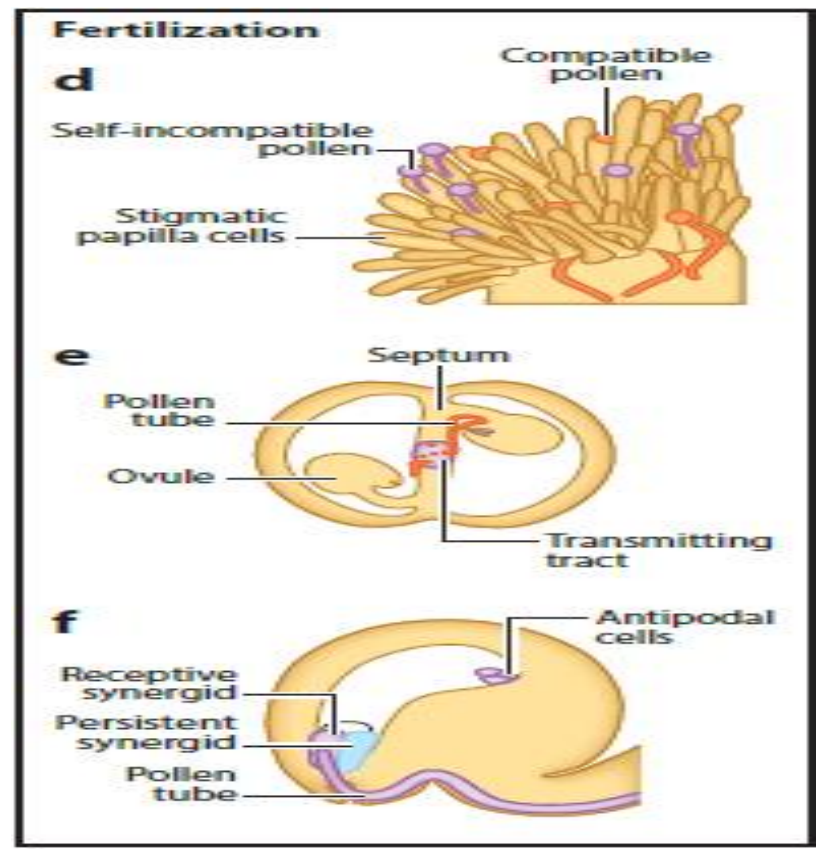

Fig.3 (d) Programmed cell death (PCD) of self-incompatible pollen. $(e)$ Transmitting tract. $(f)$ Synergid cell death and pollen tube rupture during fertilization. Dying cells or tissues are marked in purple, and dead cells or tissues are in black

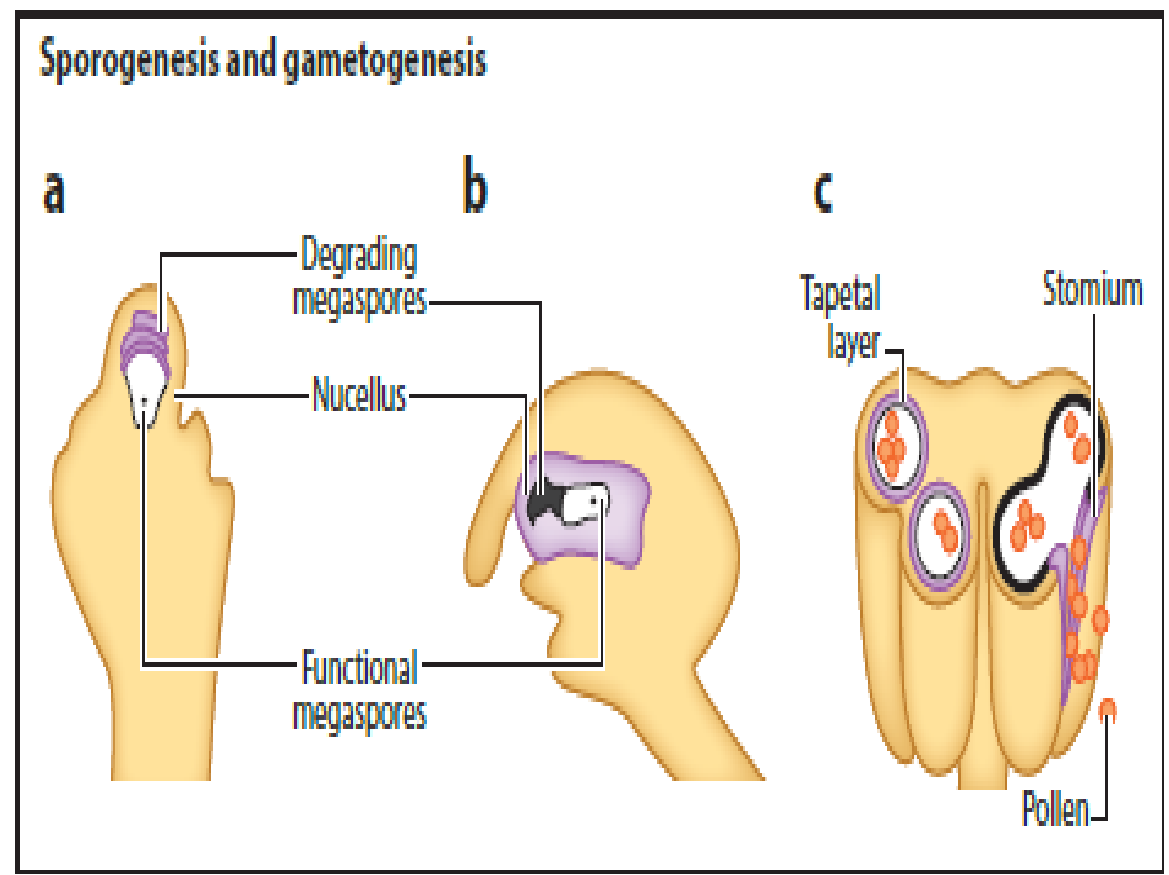


Fig.4 ( $g-j)(\mathrm{g})$-Suspensor, (h)-endosperm, (i-j) - integument and aleurone cell death during seed development and germination. Dying cells or tissues are marked in purple, and dead cells or tissues are in black

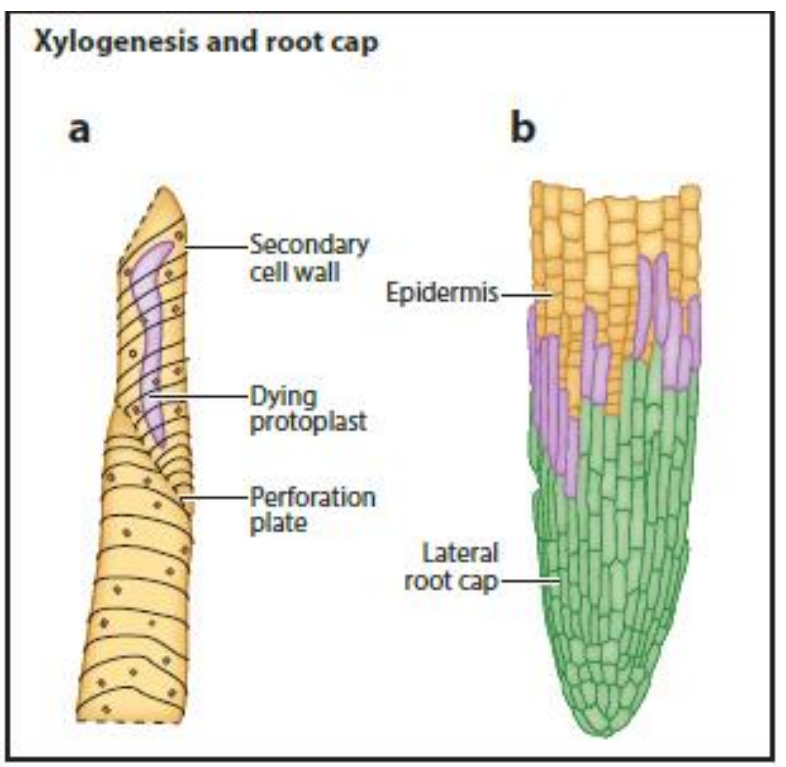

Fig.5 Cell death during vegetative development. (a) Cell death in the treachery elements. $(b)$ Programmed cell death (PCD) during root cap differentiation. Dying cells or tissues are marked in purple and dead cells or tissues are in black

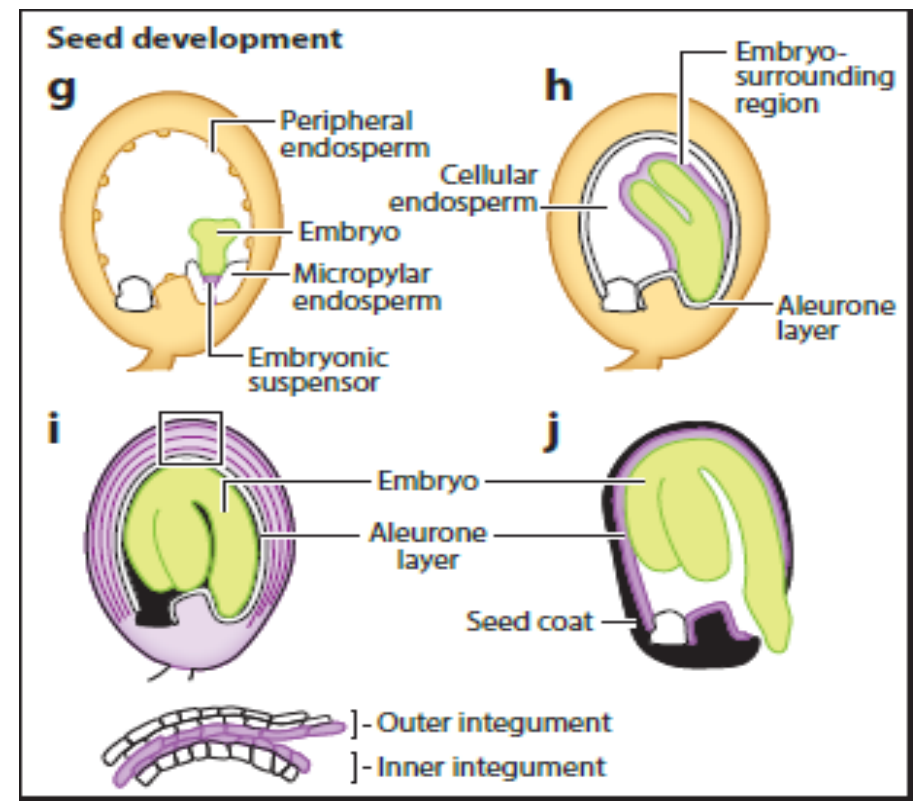


Fig.6 (c) Cell death during aerenchyma formation in rice. $(d)$ Cell death during leaf morphogenesis in Monstera deliciosa (Araceae). Dying cells or tissues are marked in purple, and dead cells or tissues are in black

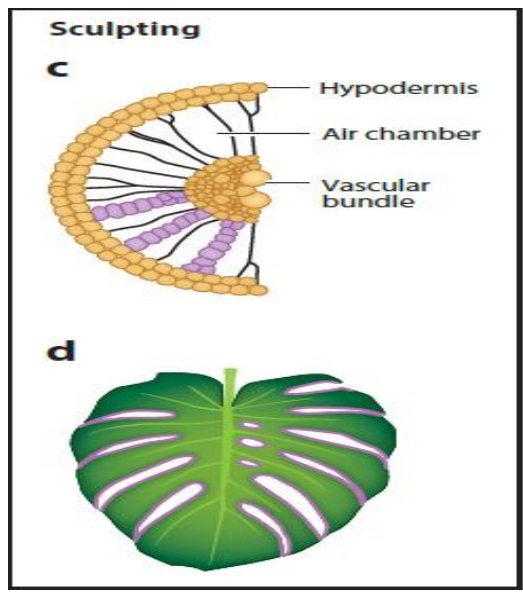

Fig.7 (e) PCD as the end point of leaf senescence. $(f)$ Floral organ senescence, dehiscence, and abscission processes during plant development. Dying cells or tissues are marked in purple

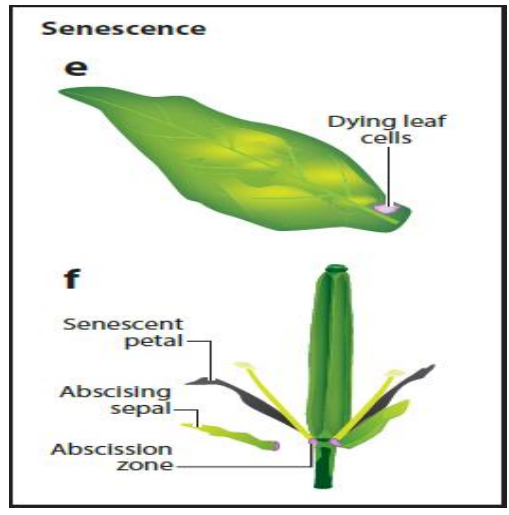

\section{References}

Bonneau, L., Ge, Y., Drury, G. E., and Gallois, P. 2008. J. Exp. Bot., 59: 491499.

Gunawardena Ahlan. 2008. Programmed cell death and tissue remodelling in plants. J. Exp. Bot. 59:445-51

Dauphinee AN, Wright H, Rantong G, Gunawardena Ahlan. 2012. The involvement of ethylene in programmed cell death and climacteric-like behaviour during the remodelling of lace plant (Aponogeton madagascariensis)

leaves.

Botany 90:1237-44

Dauphinee AN, Wright H, Rantong G, Gunawardena Ahlan. 2012. The involvement of ethylene in programmed cell death and climacteric-like behaviour during the remodelling of lace plant (Aponogeton madagascariensis) leaves. Botany 90:1237-44

Lopez-Fernandez MP, Maldonado S. 2015. Programmed cell death in seeds of angiosperms. J. Integr. Plant Biol. 57:996-1002 
Menard D, Pesquet E. 2015. Cellular interactions during tracheary elements formation and function. Curr. Opin. Plant Biol. 23:109-15

Munne-Bosch S. 2015. Senescence: Is it universal or not? Trends Plant Sci. 20:713-20

Koyama T. 2014. The roles of ethylene and transcription factors in the regulation of onset of leaf senescence. Front. Plant Sci. 5:650

Schippers JHM. 2015. Transcriptional networks in leaf senescence. Curr. Opin. Plant Biol. 27:77-83

Smertenko A, Bozhkov PV. 2014. Somatic embryogenesis: life and death processes during apical-basal patterning. J. Exp. Bot. 65: 1343-60

\section{How to cite this article:}

Dogra, S., G. Chand, B. K. Sinha and Banoo, M. 2019. Programmed Cell Death (PCD) Regulation Related to Development in Plant. Int.J.Curr.Microbiol.App.Sci. 8(12): 2324-2331. doi: https://doi.org/10.20546/ijcmas.2019.812.274 